\title{
LA-UR-16-20607
}

Approved for public release; distribution is unlimited.

Title: $\quad$ Adaptive Reconnection-based Arbitrary Lagrangian Eulerian Method

Author(s): $\quad$ Shashkov, Mikhail Jurievich

Bo, Wurigen

Intended for: Presentation at LLNL, operational visit

Issued: 
Disclaimer:

Los Alamos National Laboratory, an affirmative action/equal opportunity employer,is operated by the Los Alamos National Security, LLC for the National NuclearSecurity Administration of the U.S. Department of Energy under contract DE-AC52-06NA25396. By approving this article, the publisher recognizes that the U.S. Government retains nonexclusive, royalty-free license to publish or reproduce the published form of this contribution, or to allow others to do so, for U.S. Government purposes. Los Alamos National Laboratory requests that the publisher identify this article as work performed under the auspices of the U.S. Departmentof Energy. Los Alamos National Laboratory strongly supports academic freedom and a researcher's right to publish; as an institution, however, the Laboratory does not endorse the viewpoint of a publication or guarantee its technical correctness. 


\section{Adaptive Reconnection-based Arbitrary Lagrangian Eulerian Method A-ReALE}

Mikhail Shashkov, XCP-4, XCP Division, LANL Wurigen Bo, CCS-2, CCS Division, LANL

Currently in EXA Corporation

Thanks to

S. Sambasivan, S. Diot and S. Mao

R. Loubere, J. Breil, S. Galera, P.-H. Maire, A. Barlow, G. Ball 


\section{Outline}

- ReALE

- Adaptive Reconnection-based ALE - A-ReALE

- A-ReALE Concept

- Monitor Function, Equidistribution Principle

- Main tool: Weighted-centroidal Voronoi Tessellation

- Adaptations strategies: Maintaining spatial resolution

- How to construct weighted-centroidal Voronoi Tessellation?

- How to Compare ReALE and A-ReALE Methods?

- Numerical Examples, Sedov, triple point, shock-cavity interaction

- Conclusion and Future Work 
Standard Reconnection-based Arbitrary Lagrangian-Eulerian

\section{ReALE Methods}

- Lagrangian Phase - General polygonal meshes

- Rezone Phase

- Allows mesh reconnection Voronoi Meshes

- Mesh Smoothing - Centroidal Voronoi

- Number of Cell does not change

- Remap Phase

- Remapping between polygonal meshes with different connectivity

- Intersection-based Remap
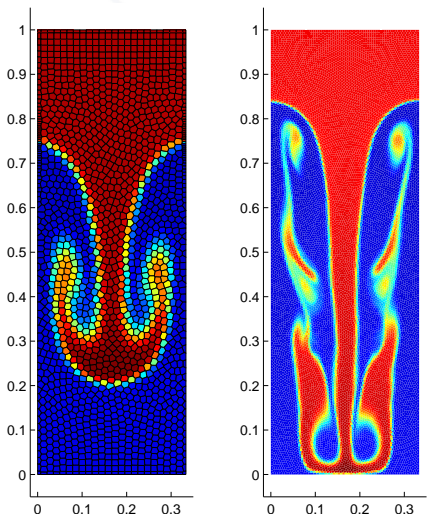
Adaptive Reconnection-based Arbitrary Lagrangian-Eulerian

\section{A-ReALE Methods}

- Lagrangian Phase - General polygonal meshes

- Rezone Phase

- Add/Remove Cells - Monitor, Equidistribution, Weighted-centroidal Voronoi

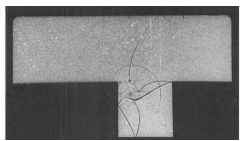

Experiment

- Mesh Reconnection - Voronoi

- Number of Cells May Change

- Remap Phase

- Remapping between polygonal meshes with different connectivity and different number of cells

- Intersection-based Remap

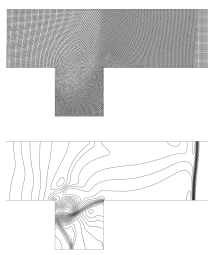

Standard ReALE
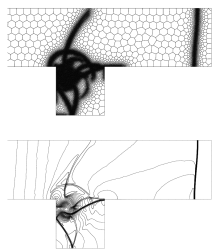

A-ReALE

Shock over Cavity 


\section{Adaptation - Design Principles}

- Monitor Function $\varphi(\mathbf{x}, t)>0$

Problem Dependent and Goal Oriented

- Interpolation Error, Error Estimation

- Feature Resolution - shock, contact, interface, boundary-layer

- Equidistribution Principle - $\int_{\Omega_{i}} \varphi(\mathbf{x}, t) d V=E(t)$-Equidistribution Level

- Adaptation strategies

- Fixed number of generators - adapting to monitor using equidistribution principle

- Maintaining constant equidistribution level during the calculation $E(t)=E\left(t_{0}\right)=$ const - number of generators changes

- Maintaining given spatial resolution where monitor reaches its max values - number of generators changes

- Main Tool - Weighted Centroidal Voronoi Meshes 


\section{Monitor Function}

- Raw Monitor - Interpolation error: $\varphi=\sqrt{\max \left(\left|\lambda_{1}\right|,\left|\lambda_{2}\right|\right)}$

Hessian $H_{u}=\left(\begin{array}{cc}\partial^{2} u / \partial x^{2} & \partial^{2} u / \partial x \partial y \\ \partial^{2} u / \partial x \partial y & \partial^{2} u / \partial y^{2}\end{array}\right)=Q^{T}\left(\begin{array}{cc}\lambda_{1} & 0 \\ 0 & \lambda_{2}\end{array}\right) Q$

- Smoothing - smoothing and removing noise

- Shape Preserving Scaling - increase min and decreases max values - to avoid very small cells - $d t$; and very big cells - accuracy

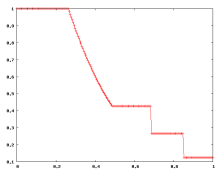

Sod problem

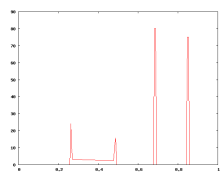

Raw Monitor

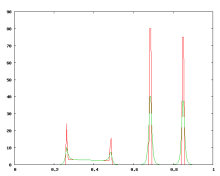

Raw \& Smoothed

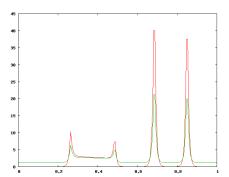

Smoothed \& Scaled 


\section{Equidistribution Principle}

- Equidistribution Principle

- Computational domain $\Omega$; Monitor function $\varphi(\mathbf{x})$

- Mesh with cells $\Omega_{i}$ satisfies equidistribution principle if

$\int_{\Omega_{i}} \varphi(\mathbf{x}) d V=$ const $=E \rightarrow \varphi_{i}\left|\Omega_{i}\right| \approx E$

- Large $\varphi_{i}$ - small $\left|\Omega_{i}\right|$

- How to construct mesh which satisfies equidistribution principle?

- Weighted centroidal Voronoi tessellation 


\section{Voronoi, Weighted-Centroidal Voronoi}

Set of generators: $\mathbf{g}_{i}=\left(x_{i}, y_{i}\right)$

Voronoi cell: $\Omega_{i}=\left\{\mathbf{r}=(x, y):\left|\mathbf{r}-\mathbf{g}_{i}\right|<\left|\mathbf{r}-\mathbf{g}_{j}\right|\right.$, for all $\left.j \neq i\right\}$

Mass centroid of the cell $(\psi(\mathbf{r})>0$ - given weight function)

$$
\mathbf{c}_{i}^{\psi}=\int_{\Omega_{i}} \mathbf{r} \psi(\mathbf{r}) d \mathbf{r} / \int_{\Omega_{i}} \psi(\mathbf{r}) d \mathbf{r},
$$

If $\mathbf{g}_{i}=\mathbf{c}_{i}^{\psi}$ - weighted-centroidal Voronoi tessellation

$$
\text { Left - Random with weight; Right - weighted-centroidal }
$$

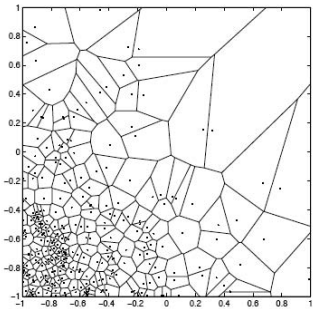

Random sampling

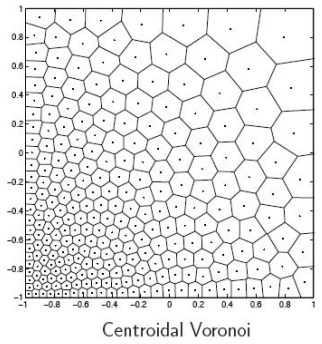

UNCLASSIFIED 


\section{Weighted-Centroidal Voronoi Tessellation}

- Properties:

- Local uniformity - spherical hexagons - accuracy of discretization

- $\psi\left(\mathbf{g}_{i}\right) h_{i}^{d+2} \approx \psi\left(\mathbf{g}_{j}\right) h_{j}^{d+2}, \quad h_{i}=2 \max _{\mathbf{r} \in \Omega_{i}}\left\|\mathbf{g}_{i}-\mathbf{r}\right\|$

$d$ dimension of the space

- How to construct mesh which satisfies equidistribution principle?

- For $d=2-\psi\left(\mathbf{g}_{i}\right) h_{i}^{4} \approx \psi\left(\mathbf{g}_{j}\right) h_{j}^{4}$

- For weighted centroidal Voronoi tessellation in 2D $\left|\Omega_{i}\right| \sim h_{i}^{2}$

$-\quad \sqrt{\psi\left(\mathbf{g}_{i}\right)}\left|\Omega_{i}\right| \approx \sqrt{\psi\left(\mathbf{g}_{j}\right)}\left|\Omega_{j}\right|$

- If we set $\psi=\varphi^{2}$, then $\varphi\left(\mathbf{g}_{i}\right)\left|\Omega_{i}\right| \approx \varphi\left(\mathbf{g}_{j}\right)\left|\Omega_{j}\right|$ - Approximate Equidistribution 


\section{Resolution and Required Number of Generators}

- If mesh satisfies equidistribution principle then

$$
\varphi\left(\mathbf{g}_{i}\right)\left|\Omega_{i}\right| \approx \varphi\left(\mathbf{g}_{j}\right)\left|\Omega_{j}\right| \approx\left(\int_{\Omega} \varphi d V\right) / N_{g}=E
$$

$N_{g}$ - number of generators, $E$ - Equid. Level

- If we want to achieve desired Equid. Level $E$ in domain $\Omega$ then we need

$$
N_{g}^{E} \approx\left(\int_{\Omega} \varphi d V\right) / E
$$

generators 
Adaptation Strategy - Maintaining Spatial Resolution

- Monitor function depends on space and time - $\varphi_{i}^{n}$

- For meshes which satisfy equidistribution $\varphi_{i}^{n} A_{i}^{n}=E^{n}$ and therefore $\varphi_{\max }^{n} A_{\min }^{n}=E^{n}$

- If we would like to maintain constant spatial resolution for in regions where monitor has its maximum values $A_{\min }^{n}=A_{\min }=$ const, then Equid. Level has to be $E^{n}=\varphi_{\max }^{n} A_{\min }$

- Number of generators need to maintain prescribed spatial resolution $A_{\text {min }}$

$$
N^{n} \approx \int_{\Omega(t)} \varphi(\mathbf{r}, t) d \mathbf{r} /\left(\varphi_{\max }^{n} A_{\min }\right)
$$




\section{How to construct weighted-centroidal Voronoi tessellation?}

- Variational formulation

- Functional

$$
\begin{aligned}
& \mathcal{K}\left(\left\{\mathbf{x}_{i}\right\}_{i=1}^{N}\right)=\sum_{i=1}^{N} \int_{\Omega_{i}} \varphi(\mathbf{x})\left\|\mathbf{x}-\mathbf{x}_{i}\right\|^{2} d \mathbf{x}, \\
& \min _{\left\{\mathbf{x}_{i}\right\}_{i=1}^{N}} \mathcal{K}\left(\left\{\mathbf{x}_{i}\right\}_{i=1}^{N}\right), \text { subject to } \mathbf{x}_{j}=\mathbf{G}_{j}, j=M+1, \ldots, N .
\end{aligned}
$$

where $\Omega_{i}$ are the Voronoi cells corresponding to the $\mathbf{x}_{i}$.

- A quasi-Newton method, the limited memory Broyden-Fletcher-Goldfarb-Shanno algorithm (L-BFGS). Need to compute Voronoi tessellation on each iteration

- Derivative

$$
\frac{\partial \mathcal{K}}{\partial \mathbf{x}_{i}}=2\left(\mathbf{x}_{i}-\mathbf{G}_{i}^{*}\right) \int_{\Omega_{i}} \varphi(\mathbf{x}) d \mathbf{x}
$$

where $\mathbf{G}_{i}^{*}$ is the mass centroid of $\Omega_{i}$. 


\section{How to construct weighted-centroidal Voronoi tessellation?}

- Initial guess - Quadtree approach

- Total \# of required generators $\Phi_{i}=\int_{\Omega_{i}^{L}} \phi(\mathbf{x}) d \mathbf{x}, \quad N_{\text {total }}^{\text {req }}=\left\lfloor\left(\sum_{i=1}^{N_{L}} \Phi_{i}\right) / E\right\rfloor$

- Number of generators required in subregion $N_{\sigma_{k}}^{\text {req }}=\left\lfloor\left(\sum_{i: G_{i} \in \sigma_{k}} \Phi_{i}\right) / E\right\rfloor$

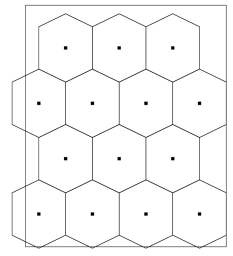

(a)

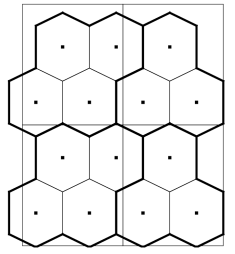

(b)

Quad-tree cells and its associated cells. The dots are the centroids of the cells.

(a) a parent quad-tree cell.

(b) The four children of the parent. The bold lines represent the boundaries of the domains associated with the children. 


\section{How to construct weighted-centroidal Voronoi tessellation?}

\section{Example}

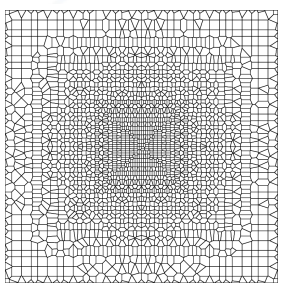

Initial guess

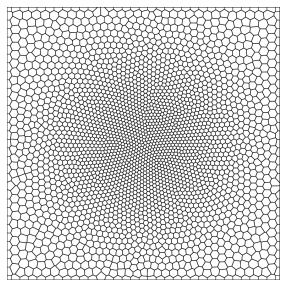

"Equidistributed mesh"

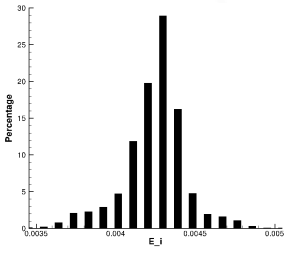

Distribution of $E_{i}$

The weight function is given by $\varphi(\mathbf{x})=\left(1+8 e^{-\|\mathbf{x}\|^{2} / 0.32}\right)^{2}$

The desired equi-distribution level $E=0.00423,2856$ generators required 


\section{How to Compare ReALE and A-ReALE Methods?}

- Choose some final time $T$ compute error (comparison can can depend on choice of $T$ )

- Number of time steps can be different for ReALE and A-ReALE

- Number of cells is changing in A-ReALE

- Error model - $C h^{q}$ ? What is $h$ ? For A-ReALE we can use $h=\sqrt{A_{\min }}$; for ReALE we only can choose $N^{-1 / 2}$ (for given initial mesh). Small parameter has different meaning and we cannot compare convergence rates.

- Error strongly depends on choice of initial mesh for ReALE

- Our approach - run A-ReALE, compute error and then choose number of generators in ReALE such that error is approximately the same.

- Compare total number of generators used in "space-time".

- One can use number of cells in the "equivalent" uniform mesh - mesh in which all cells have the same area equal to $A_{\min }-N_{\text {eq }}=A / A_{\min }$ 


\section{Sedov Problem - ReALE vs. A-ReALE-Meshes}

Meshes for ReALE - 4000 and A-ReALE - $A_{\min }=1.6 \times 10^{-4}$ - the same final $L_{1}$ error
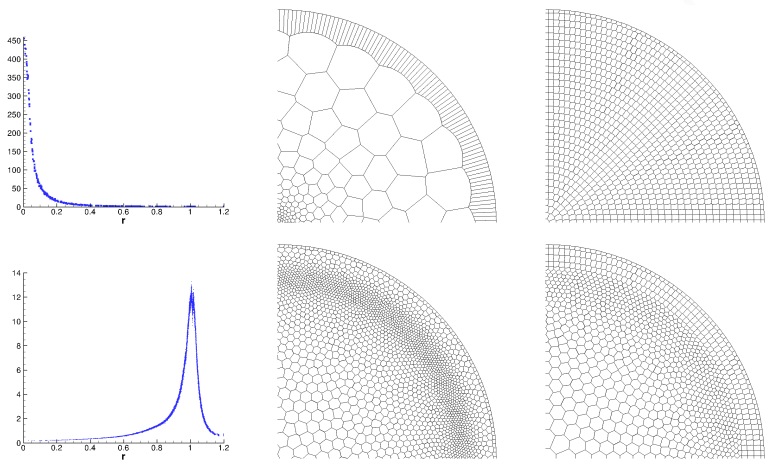

Monitor

A-ReALE

ReALE 


\section{Sedov Problem - ReALE vs. A-ReALE-Symmetry}
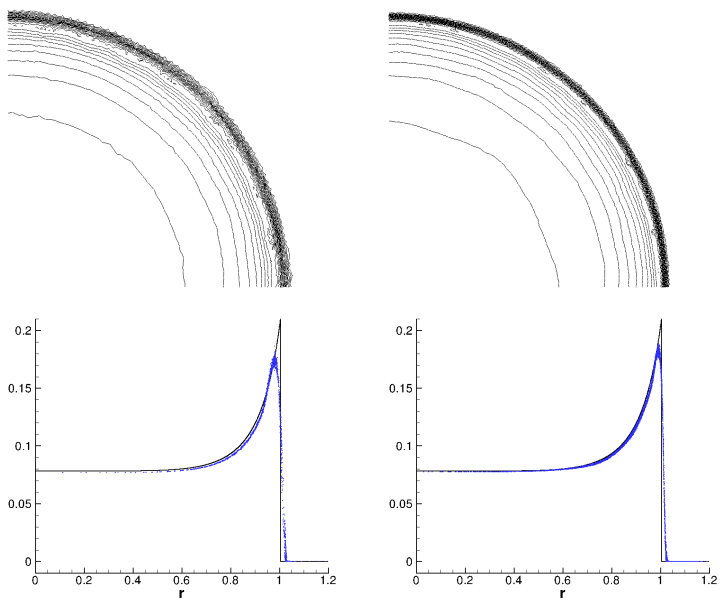

ReALE UNCLASSIFIED

A-ReALE 


\section{Sedov Problem - ReALE vs. A-ReALE-Symmetry}
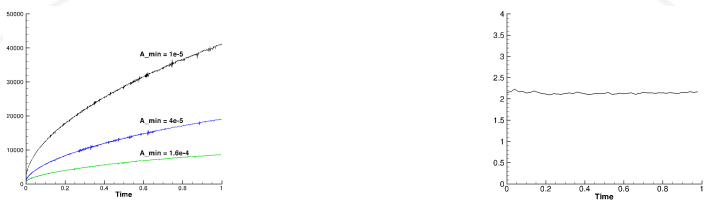

A-ReALE - \# of gen. ratio of the \# number of generators -finest meshes.
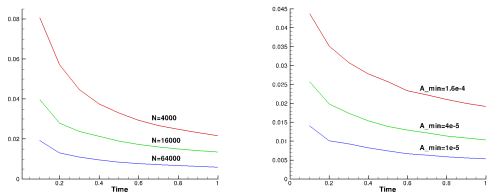

ReALE

A-ReALE

L1 density error vs. time - first order convergence - different $h$ 
Sedov Problem - ReALE vs. A-ReALE-Convergence

\begin{tabular}{|ccc|}
\hline$A_{\min }$ & $e(1)$ & $q(1)$ \\
\hline $1.6 \mathrm{e}-4$ & $1.92016 \mathrm{e}-02$ & \\
$4 \mathrm{e}-5$ & $1.02941 \mathrm{e}-02$ & 0.89941 \\
$1 \mathrm{e}-5$ & $5.21725 \mathrm{e}-03$ & 0.98046 \\
\hline \multicolumn{4}{|c|}{ L1 pressure error for A-ReALE }
\end{tabular}

\begin{tabular}{|ccc|}
\hline$N$ & $e(1)$ & $q(1)$ \\
\hline 4000 & $2.15568 \mathrm{e}-02$ & \\
16000 & $1.34996 \mathrm{e}-02$ & 0.675 \\
64000 & $5.85117 \mathrm{e}-03$ & 1.206 \\
\hline \multicolumn{4}{|c}{ L1 pressure error for ReALE }
\end{tabular}

UNCLASSIFIED 
Sedov Problem - ReALE vs. A-ReALE-Comparison

\begin{tabular}{|c|cccc|}
\hline & $e(1)$ & $N(1)$ & $N_{s t}(1)$ & time steps \\
\hline ReALE & $5.85117 \mathrm{e}-03$ & 64000 & $9.73 \mathrm{e} 7$ & 1716 \\
A-ReALE & $5.21725 \mathrm{e}-03$ & 40970 & $4.43 \mathrm{e} 7$ & 2107 \\
\hline
\end{tabular}
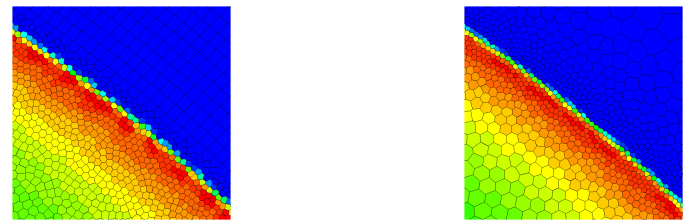

ReALE, 64000 generators A-ReALE, $A_{\min }=10^{-5}$

UNCLASSIFIED 


\section{Sedov Problem - ReALE vs. A-ReALE-Comparison}
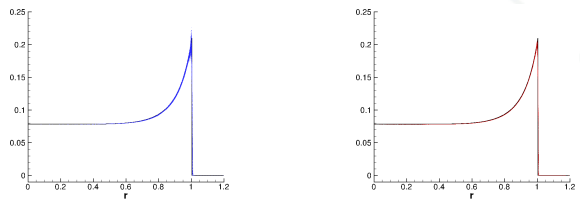

ReALE, 64000 generators A-ReALE, $A_{\min }=10^{-5}$

The scatter plot of pressure along the radial direction - high resolution

\begin{tabular}{|cccc|}
\hline$A_{\min }$ & $N_{e q}$ & $N(1)$ & $N_{e q} / N(1)$ \\
\hline $1.6 \mathrm{e}-4$ & 28274 & 8557 & 3.3042 \\
$4 \mathrm{e}-5$ & 113096 & 19151 & 5.9055 \\
$1 \mathrm{e}-5$ & 452384 & 40970 & 11.042 \\
\hline
\end{tabular}




\section{Triple Point Problem - ReALE vs. A-ReALE}

Approximately the same number number of generators in space-time

$$
N_{R e A L E}=\sim 34000, A_{\min }^{A-R e A L E}=5 \times 10^{-5}
$$

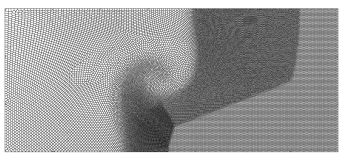

ReALE - Mesh, $N_{g}=33556$

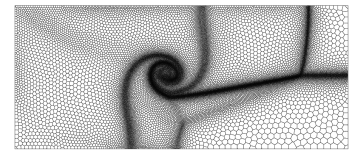

A-ReALE - Mesh, $N_{g}=24374$

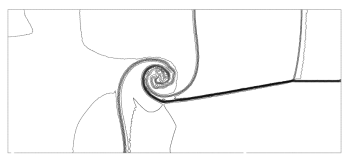

ReALE - IE

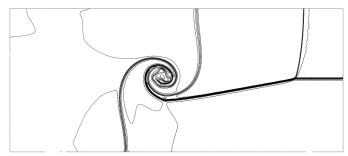

A-ReALE - IE

$$
t=3
$$




\section{Triple Point Problem - ReALE vs. A-ReALE}
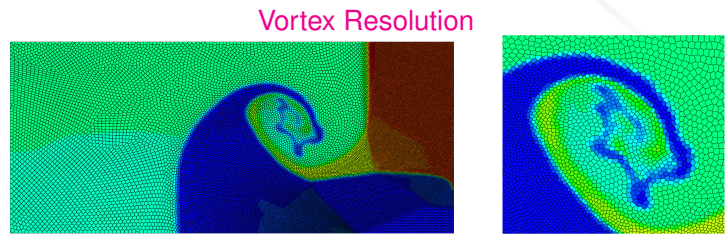

\section{ReALE}
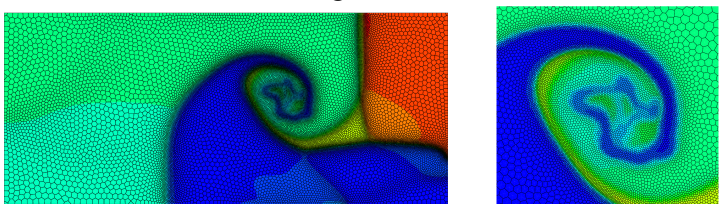

$$
\begin{gathered}
\text { A-ReALE } \\
t=5
\end{gathered}
$$




\section{Interaction between a shock and cavity}

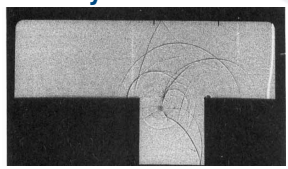

The shadowgraph from the experimental results - Igra et al.
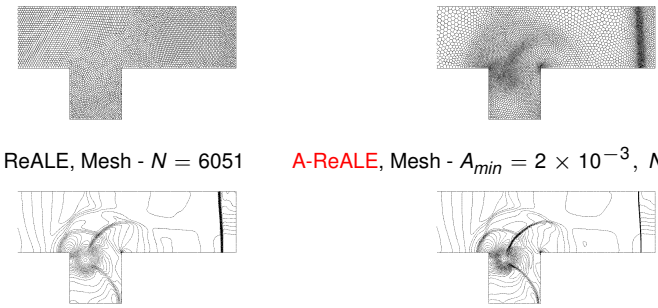

A-ReALE, Mesh $-A_{\min }=2 \times 10^{-3}, N(420)=6450$

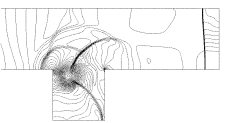

ReALE, $N=39593$

A-ReALE, $A_{\min }=1.25 \times 10^{-4}, N(420)=33179$

\begin{tabular}{|c|ccc|}
\hline & $N(420)$ & $N_{\text {st }}(420)$ & time steps \\
\hline ReALE $N=39593$ & 39593 & 159559790 & 4030 \\
A-ReALE $A_{\min }=1.25 \times 10^{-4}$ & 33179 & 177856789 & 7086 \\
\hline
\end{tabular}




\section{ShaPo-Voronoi Mesh Generator}

Contract with Kitware SAS (Lyon, France) - Joachim Pouderoux LANL - M. Shashkov, M. Kenamond, M. Berndt, B. Jean, D. Herring ShaPo-2D Main Features

- 2D Voronoi tessellation from a user provided set of generators within a closed domain

- Domain boundary: a polygon that can be

- Convex

- Simply connected

- Non-convex

- Multiply-connected - with or without holes

- Provides full connectivity of the generated mesh along with other useful information

- Points, edges \& cells connectivity - using winged edges

- Input boundary edge a mesh edge stands on, new points introduced on a boundary edge, etc.

- Can merge small edges

- Can perform mesh relaxation Centroidal Voronoi

- Mesh export and visualization functions

- Remeshing layer to remesh part of existing mesh 


\section{ShaPo, ReALE and FLAG}

\section{LANL SETUP Project - B. Jean, D. Herring}
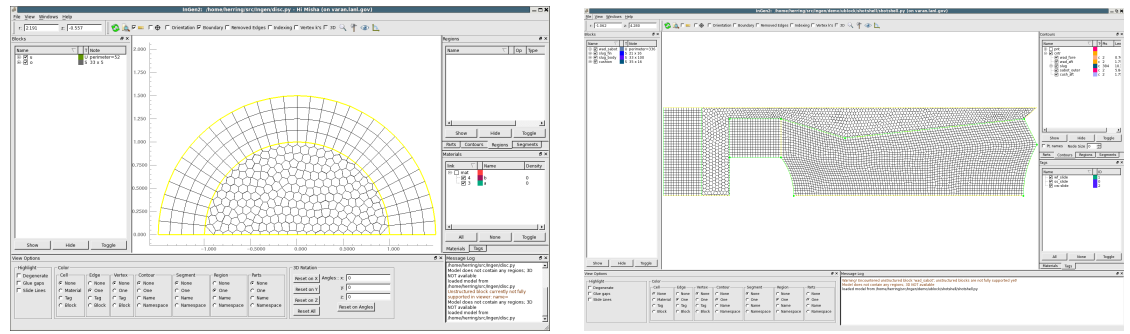

UNCLASSIFIED 


\section{Using ReALE in Combination with Lagrangian and ALE Methods}

Personal Communication - A.Barlow (AWE) and G.Ball (AWE)
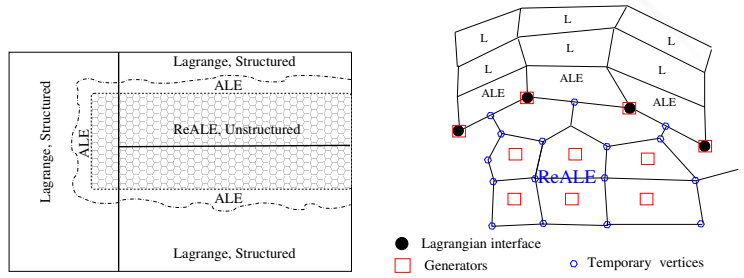

- Predetermined regions with Lagrangian boundaries with ReALE inside

- Dynamically changing regions with Lagrangian boundaries with ReALE inside

- ALE buffer region 


\section{ShaPo-3D Development}
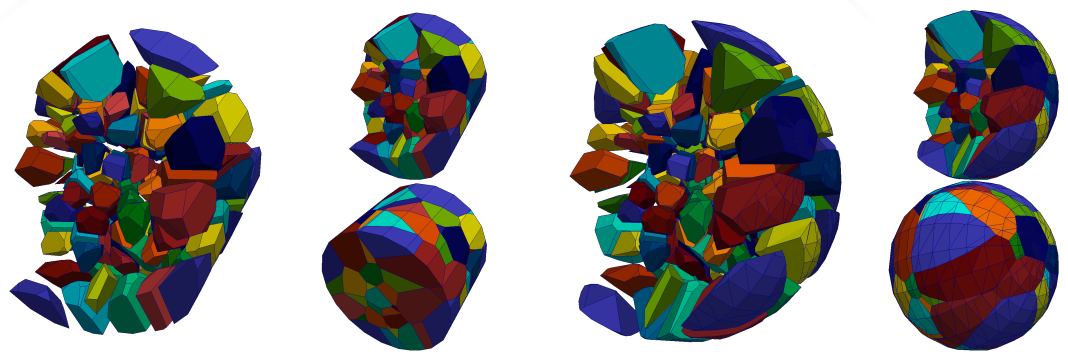


\section{Conclusion and Future Work}

- Developed new adaptive reconnection based framework

- Demonstrated A-ReALE performance on series of examples

- Papers:

- W. Bo, M. Shashkov, Adaptive reconnection-based arbitrary

Lagrangian Eulerian method, J. of Comput. Phys. 299 (2015) 902-939

- W. Bo, M. Shashkov, textcolorredR-Adaptive Reconnection-based Arbitrary Lagrangian Eulerian method R-ReALE, J.Math. Study 48(2) (2015) 125-167.

- Implementation in FLAG - ongoing 


\section{Thanks}

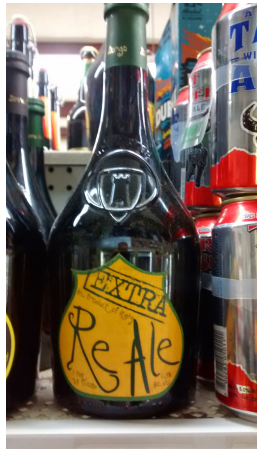

Picture by M. Berndt (LANL) from Kokoman Pojoaque Valley, NM Liquor Store

Papers, Reports: https://www.researchgate.net/profile/Mikhail_Shashkov 